\title{
Characteristics and management of pain in patients with Klippel-Feil syndrome: analysis of a global patient-reported registry
}

\author{
Kishan Patel, BS, ${ }^{1}$ Hardy Evans, BA, ${ }^{2}$ Samuel Sommaruga, MD, ${ }^{3}$ Pia Vayssiere, MD, ${ }^{3}$ \\ Tariq Qureshi, MBBS, MPH, ${ }^{1}$ Luis Kolb, MD, ${ }^{1}$ Michael G. Fehlings, MD, PhD, ${ }^{4}$ \\ Joseph S. Cheng, MD, MS, ${ }^{2}$ Enrico Tessitore, MD, ${ }^{3}$ Karl Schaller, MD, ${ }^{3}$ and Aria Nouri, MD, MSc ${ }^{3}$
}

\begin{abstract}
1Department of Neurosurgery, Yale University School of Medicine, New Haven, Connecticut; 'Department of Neurosurgery, University of Cincinnati College of Medicine, Cincinnati, Ohio; ${ }^{3}$ Department of Neurosurgery, Geneva University Hospitals, Geneva, Switzerland; and ${ }^{4}$ Department of Neurosurgery, University of Toronto, Toronto, Ontario, Canada
\end{abstract}

\begin{abstract}
OBJECTIVE Klippel-Feil syndrome (KFS) is characterized by congenital fusion of the cervical vertebrae. Due to its rarity, minimal research has been done to assess the quality and management of pain associated with this disorder. Using a large global database, the authors report a detailed analysis of the type, location, and treatment of pain in patients with KFS.
\end{abstract}

METHODS Data were obtained from the Coordination of Rare Diseases at Stanford registry and Klippel-Feil Syndrome Freedom registry. The cervical fusions were categorized into Samartzis type I, II, or III. The independent-sample t-test, Wilcoxon rank-sum test, and Friedman test were conducted, with significance set at $p<0.05$.

RESULTS Seventy-five patients (60 female, 14 male, and 1 unknown) were identified and classified as having the following types of Samartzis fusion: type I, $n=21$ (28\%); type II, $n=15$ (20\%); type III, $n=39(52 \%)$. Seventy participants (93.3\%) experienced pain associated with their KFS. The median age of patients at pain onset was 16.0 years (IQR 6.75-24.0 years), and the median age when pain worsened was 28.0 years (IQR 15.25-41.5 years). Muscle, joint, and nerve pain was primarily located in the shoulders/upper back (76\%), neck (72\%), and back of head $(50.7 \%)$ and was characterized as tightness (73\%), dull/aching (67\%), and tingling/pins and needles (49\%). Type III fusions were significantly associated with greater nerve pain $(p=0.02)$, headache/migraine pain $(p=0.02)$, and joint pain $(p=0.03)$ compared to other types of fusion. Patients with cervical fusions in the middle region (C2-6) tended to report greater muscle, joint, and nerve pain $(p=0.06)$. Participants rated the effectiveness of oral over-the-counter medications as 3 of 5 (IQR 1-3), oral prescribed medications as 3 of 5 (IQR 2-4), injections as 2 of 5 (IQR 1-4), and surgery as 3 of 5 (IQR 1-4), with 0 indicating the least pain relief and 5 the most pain relief. Participants who pursued surgical treatment reported significantly more comorbidities $(p=0.02)$ and neurological symptoms $(p=0.01)$ than nonsurgically treated participants and were significantly older when pain worsened $(p=0.03)$, but there was no difference in levels of muscle, joint, or nerve pain $(p=0.32)$; headache/migraine pain $(p=0.35)$; total number of cervical fusions ( $p=0.77)$; location of fusions; or age at pain onset $(p=0.16)$.

CONCLUSIONS More than $90 \%$ of participants experienced pain. Participants with an increased number of overall cervical fusions or multilevel, contiguous fusions reported greater levels of muscle, joint, and nerve pain. Participants who pursued surgery had more comorbidities and neurological symptoms, such as balance and gait disturbances, but did not report more significant pain than nonsurgically treated participants.

https://thejns.org/doi/abs/10.3171/2019.9.SPINE19820

KEYWORDS Klippel-Feil syndrome; pain; congenital; cervical spine; cervical fusion

$\mathrm{K}$ LIPPEL-FEIL syndrome (KFS) is a rare congenital disorder characterized by fusion of the cervical vertebrae. Classically, patients with KFS exhibit a triad of low posterior hairline, a short neck, and restricted mobility of the upper spine, although only $50 \%$ of cases include all 3 elements. The prevalence of KFS has been estimated to be $0.71 \%$ based on cadaver bones, ${ }^{2}$ and a recent study using CT scans in an emergency department corroborated this finding, reporting a prevalence of $0.58 \% .{ }^{4} \mathrm{KFS}$ is known to have a slight female preponderance. ${ }^{1}$ Although 
the etiology is unknown, KFS is thought to be caused by faulty segmentation during early embryological development and has been associated with mutations in homeobox genes and differentiation factors, including $M E O X 1$,

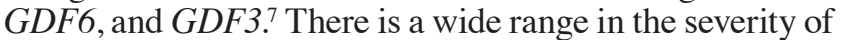
symptoms; some pediatric patients are asymptomatic and may transition into adulthood unaware of their condition, while other individuals experience significant symptoms, including spinal stenosis, instability, degenerative cervical myelopathy, scoliosis, and Sprengel deformity. ${ }^{9}$

Pain associated with KFS can be significant but is not commonly reported in the literature. As a result, there is a gap in our understanding of the most common types and locations of pain in KFS, as well as in the treatment modalities that offer the greatest therapeutic or palliative benefit. In this study, we use data from a rare disease registry compiled by two nonprofit organizations-representing one of the largest collections of KFS patient data to date - to characterize the quality and management of pain experienced in KFS.

\section{Methods}

The patient data used in the present study were derived from two surveys compiled by two nonprofit organizations: Sanford Health's Coordination of Rare Diseases at Sanford (CoRDS) registry, which collects information about rare diseases (Appendix 1), and Klippel-Feil Syndrome Freedom, which compiles data specifically about KFS (Appendix 2). Information about these surveys is described in a previously published study. ${ }^{10}$ In total, 75 patients with fusions of the cervical vertebrae were identified. These cases were categorized into the following fusion types using Samartzis criteria: type I, a single fusion; type II, multiple noncontiguous fusions; and type III, multiple contiguous fusions. ${ }^{12}$ The cervical fusions were also categorized into those of the upper level (occiput-C1, C1-2), which approximates the occipitocervical junction, middle level (C2-3, C3-4, C4-5, C5-6), and lower level (C6-7, C7-T1), which approximates the cervicothoracic junction.

In these surveys, participants were asked to describe the quality of their pain, with 0 being minimal pain and 10 being severe pain, and to rate the effectiveness of various pain relief modalities, with 0 being no pain relief and 5 being maximal pain relief. Examples that were provided in the survey for each general treatment modality included "Tylenol, ibuprofen" for over-the-counter oral medications, "Lyrica, valium" for prescribed oral medications, "trigger point injections, facet blocks, epidural injections, nerve blocks" for injections, and "fusion, laminectomy, discectomy" for surgery, although these examples were not exhaustive.

Variables with normal distributions were reported as the mean $\pm \mathrm{SD}$, while variables with nonnormal distributions were reported as the median (IQR). An independent sample t-test or Wilcoxon rank-sum test (for nonnormal data) was used to determine the association between the location and severity of cervical fusion with various types of pain, as well as the characteristics of patients who underwent surgical management. The Friedman test was
TABLE 1. Summary of demographic data and Samartzis classification in 75 patients with KFS

\begin{tabular}{lc}
\hline \multicolumn{1}{c}{ Demographics } & Value \\
\hline Median age (IQR), yrs & \\
\hline When symptoms began & $16.0(6.75-24.0)$ \\
\hline When symptoms worsened & $28.0(15.25-41.5)$ \\
\hline Sex & $18.7 \%(n=14)$ \\
\hline Male & $80.0 \%(n=60)$ \\
\hline Female & $1.3 \%(n=1)$ \\
\hline Did not respond & \\
\hline Samartzis classification & $28.0 \%(n=21)$ \\
\hline Type I (single-level fusion) & $20.0 \%(n=15)$ \\
\hline Type II (multiple noncontiguous fusions) & $52.0 \%(n=39)$ \\
\hline Type III (multiple contiguous fusions) & \\
\hline Level of fusion & $22.7 \%(n=17)$ \\
\hline Occiput-C1 & $32.0 \%(n=24)$ \\
\hline C1-2 & $56.0 \%(n=42)$ \\
\hline C2-3 & $40.0 \%(n=30)$ \\
\hline C3-4 & $56.0 \%(n=42)$ \\
\hline C4-5 & $42.7 \%(n=32)$ \\
\hline C5-6 & $33.3 \%(n=25)$ \\
\hline C6-7 & $17.3 \%(n=13)$ \\
\hline C7-T1 & $41.3 \%(n=31)$ \\
\hline Upper level (occiput-C1, C1-2) & $93.3 \%(n=70)$ \\
\hline Middle level (C2-3, C3-4, C4-5, C5-6) & $38.7 \%(n=29)$ \\
\hline Lower level (C6-7, C7-T1) & \\
\hline
\end{tabular}

Except for those related to age, values are expressed as the percentage (number) of patients.

used to determine the effectiveness of various treatment modalities, and post hoc analysis with a Wilcoxon signedrank test was conducted using Bonferroni correction for multiple comparisons. The Spearman correlation coefficient was used to determine the association between the overall level of pain and the number of fusions, average number of comorbidities, age at symptom onset, and age at symptom worsening. Significance was considered at $\mathrm{p}<$ 0.05 , and trends were considered at $\mathrm{p}<0.10$.

\section{Results}

A total of 75 patients were identified with congenital cervical fusions. The demographic details of these individuals have been described in a prior report..$^{10}$ Based on the Samartzis classification, $28 \%$ of patients had type I (single-level fusion), 20\% had type II (multiple noncontiguous fusions), and $52 \%$ had type III (multiple contiguous fusions) disease. Approximately $41 \%$ of participants identified a fusion in the upper level, compared to $93 \%$ in the middle level and 39\% in the lower level (Table 1).

Pain was reported in $93.3 \%$ of participants. The most common location of pain associated with KFS was in the shoulders and upper back $(76 \%)$, followed by the neck (72\%), back of the head (51\%), middle back (28\%), and lower back $(28 \%)$. There was a relatively even distribution 
with regard to the onset of pain, with pain appearing gradually in $27 \%$ of participants, appearing gradually but suddenly worsening in $20 \%$, and appearing suddenly in $23 \%$ of the cohort. The pain was most commonly described as constant (52\%), although approximately $32 \%$ of the participants described less-frequent pain that ranged from rare to intermittent. There were a wide variety of descriptions of the quality of KFS pain, although tightness was most commonly reported (73\%), followed by dull/aching (67\%) and tingling/pins and needles (49\%) (Table 2).

Participants with multiple, contiguous fusions (Samartzis type III) reported significantly greater overall pain $(\mathrm{p}=0.02)$, nerve pain $(\mathrm{p}=0.02)$, headache/migraine pain $(\mathrm{p}=0.02)$, and joint pain $(\mathrm{p}=0.03)$ than patients with other fusion types. There was no significant difference in the level of muscle tension, soreness, or spasm $(\mathrm{p}=0.86)$ (Table 3 ). There was a statistically significant positive correlation between the number of fusions and the level of overall muscle, joint, and nerve pain $(\mathrm{p}=0.02)$. There was no association between overall muscle, joint, and nerve pain and other demographic factors, such as sex $(p=0.20)$, age at symptom onset $(\mathrm{p}=0.35)$, age at symptom worsening $(p=0.24)$, or average number of comorbidities $(p=$ 0.28 ). Compared to others, participants with middle-level cervical fusions tended to report greater overall muscle, joint, and nerve pain $(\mathrm{p}=0.06)$, although this did not reach statistical significance (Table 4).

With regard to pain management, participants rated the effectiveness of oral over-the-counter medications as 3 of 5 (IQR 1-3), prescribed oral medications as 3 of 5 (IQR 2-4), injections as 2 of 5 (IQR 1-4), and surgical correction as 3 of 5 (IQR 1-4). The Friedman test was completed using data from 18 participants who had used all 4 treatment modalities, which demonstrated a statistically significant difference $(\mathrm{p}=0.002)$. Pairwise comparison with Bonferroni correction (significance at $\mathrm{p}<$ 0.0083 ) showed that prescribed oral medications provided significantly more relief than over-the-counter medications $(p=0.003)$, but there was no significant difference between prescribed oral medications and injections $(\mathrm{p}=$ $0.015)$ or surgery $(\mathrm{p}=0.087)$. Participants who pursued surgical treatment reported significantly more comorbidities $(p=0.02)$ and neurological symptoms $(p=0.01)$ than nonsurgically treated participants and were significantly older when pain worsened ( $p=0.03)$, but there was no difference in levels of muscle, joint, or nerve pain $(p=0.32)$, headache/migraine pain ( $\mathrm{p}=0.35)$, total number of cervical fusions $(\mathrm{p}=0.77)$, location of fusions, or age at pain onset $(\mathrm{p}=0.16)($ Table 5).

\section{Discussion}

The existing literature surrounding KFS is mainly limited to case reports and case series, which are skewed toward unique or more-severe presentations of illness. Indeed, our data set also contains more-severe presentations than have been previously reported; $72 \%$ of our cohort had either Samartzis type II or III fusions. In contrast, previous studies, such as that by Gruber et al., reported exclusively on type I fusions in the 17 cases that were identified out of 2917 patients. ${ }^{4}$ This trend likely reflects the fact that
TABLE 2. Location, onset, frequency, and quality of pain in 75 patients with KFS

\begin{tabular}{|c|c|}
\hline Variable & $\%$ (no.) of Patients \\
\hline \multicolumn{2}{|l|}{ Presence of pain } \\
\hline Yes & $93.3 \%(n=70)$ \\
\hline No & $2.7 \%(n=2)$ \\
\hline Did not respond & $4.0 \%(n=3)$ \\
\hline \multicolumn{2}{|l|}{ Location of pain } \\
\hline Shoulders/upper back & $76.0 \%(n=57)$ \\
\hline Neck & $72.0 \%(n=54)$ \\
\hline Back of head & $50.7 \%(n=38)$ \\
\hline Middle back & $28.0 \%(n=21)$ \\
\hline Lower back & $28.0 \%(n=21)$ \\
\hline Front of head (face/jaw/ears) & $21.3 \%(n=16)$ \\
\hline Hands & $20.0 \%(n=15)$ \\
\hline Hips \&/or pelvis & $17.3 \%(n=13)$ \\
\hline Upper arm & $17.3 \%(n=13)$ \\
\hline Lower arm & $14.7 \%(n=11)$ \\
\hline Lower leg & $9.3 \%(n=7)$ \\
\hline Buttocks & $8.0 \%(n=6)$ \\
\hline Feet & $6.7 \%(n=5)$ \\
\hline \multicolumn{2}{|l|}{ Onset of pain } \\
\hline Gradually appeared & $26.7 \%(n=20)$ \\
\hline Gradually appeared but worsened suddenly & $20.0 \%(n=15)$ \\
\hline Suddenly appeared & $22.7 \%(n=17)$ \\
\hline Did not respond & $30.7 \%(n=23)$ \\
\hline \multicolumn{2}{|l|}{ Frequency of muscle, joint, \& nerve pain } \\
\hline Constant & $52.0 \%(n=39)$ \\
\hline Intermittent & $22.7 \%(n=17)$ \\
\hline Occasional & $6.7 \%(n=5)$ \\
\hline Rare & $2.7 \%(n=2)$ \\
\hline Varies & $8.0 \%(n=6)$ \\
\hline Did not respond & $8.0 \%(n=6)$ \\
\hline \multicolumn{2}{|l|}{ Quality of pain } \\
\hline Cracking/popping & $36.0 \%(n=27)$ \\
\hline Cramping/squeezing & $34.7 \%(n=26)$ \\
\hline Dull/aching & $66.7 \%(n=50)$ \\
\hline Hot/burning & $32.0 \%(n=24)$ \\
\hline Numbness & $40.0 \%(n=30)$ \\
\hline Shooting & $30.7 \%(n=23)$ \\
\hline Spasms & $41.3 \%(n=31)$ \\
\hline Stabbing/sharp & $42.7 \%(n=32)$ \\
\hline Throbbing & $46.7 \%(n=35)$ \\
\hline Tightness & $73.3 \%(n=55)$ \\
\hline Tingling/pins \& needles & $49.3 \%(n=37)$ \\
\hline Weakness & $40.0 \%(n=30)$ \\
\hline
\end{tabular}

many patients with KFS are asymptomatic and remain unaware of their illness in the absence of spinal imaging or frank physical deformity. As suggested by the degree of multiple fusions in our cohort, the patients who were 
TABLE 3. Association between Samartzis classification and the severity of muscle, joint, nerve, and headache pain

\begin{tabular}{lccc}
\hline \multirow{2}{*}{ Type of Pain* } & \multicolumn{2}{c}{ Samartzis Type } & $\begin{array}{c}p \\
\text { Value }\end{array}$ \\
\cline { 2 - 3 } $\begin{array}{l}\text { Overall muscle, joint, nerve } \\
\text { pain }\end{array}$ & $6.0(3.75-7.0)$ & $7.0(5.0-8.0)$ & $\mathbf{0 . 0 2}$ \\
\hline $\begin{array}{l}\text { Nerve pain } \\
\text { Muscle tension, soreness, } \\
\text { spasm }\end{array}$ & $7.0(3.0-7.0)$ & $7.0(6.0-8.0)$ & $\mathbf{0 . 0 2}$ \\
\hline Headache, migraine pain & $6.0(4.0-8.25)$ & $7.0(5.0-8.0)$ & 0.86 \\
\hline Joint pain & $6.0(5.0-8.0)$ & $7.0(5.0-9.0)$ & $\mathbf{0 . 0 3}$ \\
\hline
\end{tabular}

Boldface type indicates statistical significance.

* Pain was rated on a scale from 1 to 10 , with 10 representing the most severe pain.

included in the Klippel-Feil Syndrome Freedom registry likely suffered from more-severe presentations and, therefore, were more likely to become aware of their illness at an earlier age and subsequently connect with nonprofit organizations.

Prior studies of KFS have suggested a slightly increased prevalence in females, with estimates ranging from $57 \%$ to $70 \% .^{12}$ Our data also show a significant majority of females $(80 \%)$, although this proportion is higher than has been previously reported in the literature. The reasons behind this are unclear-the higher prevalence may represent a true epidemiological phenomenon in which women have a genetic or physiological predisposition to more-severe forms of KFS, or it may reflect an increased tendency for women to connect with and participate in rare disease registries compared to men. Further work is required to elucidate the female bias of this disease.

The experience of pain in KFS has not been well reported in the literature. The closest analog to the assessment of pain severity in KFS is the use of the Neck Disability Index (NDI) in prior studies. Nouri et al., for instance, showed that KFS patients exhibited a weak trend toward lower NDI scores, reflecting less disability compared to patients with cervical myelopathy without cervical fusion, while Cho et al. showed no significant difference in preoperative NDI scores between KFS patients and matched controls. ${ }^{3,9}$ However, it is important to note that pain severity makes up only $10 \%$ of the NDI, and it is therefore difficult to interpret NDI as a direct measure of pain. In our study, an overwhelming majority of participants (approximately 93\%) indicated muscle, joint, or nerve pain associated with KFS, and over $50 \%$ of participants stated that this pain was constant. Although this may reflect that more-severe presentations of KFS are represented in our cohort, it also suggests that in these syndromic patients, pain is a significant and inadequately studied feature of their illness.

Our results show that the majority of pain in KFS patients is located in the upper back, neck, and back of the head, as would be expected from a cervical-level fusion. However, there is still a significant amount of pain reported in atypical areas, including the lower back, hip/ pelvis, and lower extremities. It is difficult to discern

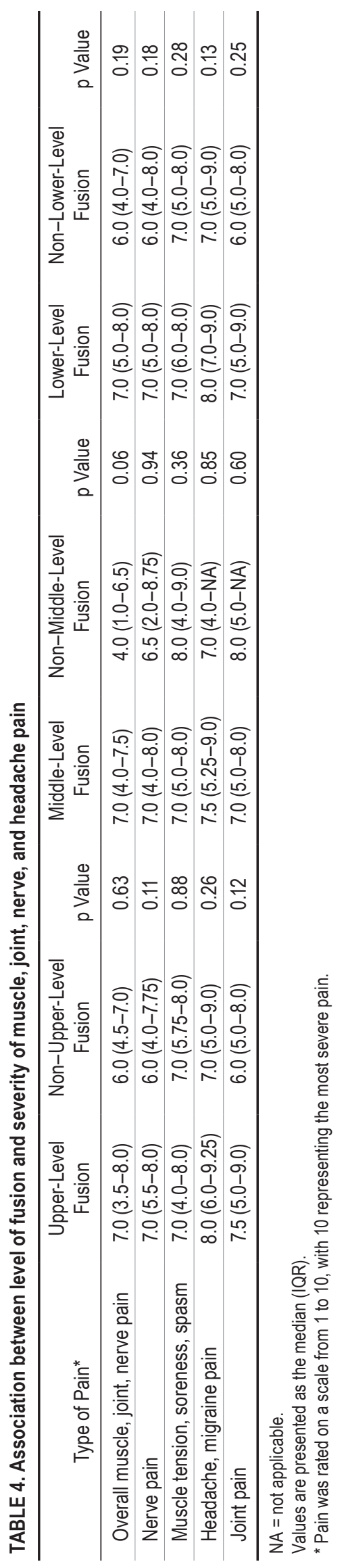

J Neurosurg Spine Volume 32 • April 2020 
TABLE 5. Characteristics of patients who underwent surgical and nonsurgical management to treat their KFS symptoms

\begin{tabular}{lccc}
\hline \multicolumn{1}{c}{ Variable } & $\begin{array}{c}\text { Surgical } \\
\text { Management }\end{array}$ & $\begin{array}{c}\text { Nonsurgical } \\
\text { Management }\end{array}$ & $\begin{array}{c}\mathrm{p} \\
\text { Value }\end{array}$ \\
\hline $\begin{array}{c}\text { Age at symptom } \\
\text { onset, yrs }\end{array}$ & $18.5(10.5-23.75)$ & $11.5(5.0-24.0)$ & 0.16 \\
\hline $\begin{array}{c}\text { Age at symptom } \\
\text { worsening, yrs }\end{array}$ & $34.0(23.5-43.0)$ & $19.0(11.0-33.0)$ & 0.03 \\
\hline $\begin{array}{c}\text { Total no. of cervical } \\
\text { fusions }\end{array}$ & $2.0(1.0-5.0)$ & $3.0(2.0-4.0)$ & 0.77 \\
\hline $\begin{array}{c}\text { No. of comorbidities } \\
\text { No. of neurological } \\
\text { symptoms }\end{array}$ & $14.9 \pm 8.45$ & $11.1 \pm 5.87$ & 0.02 \\
\hline $\begin{array}{c}\text { Overall muscle, joint, } \\
\& \text { nerve pain* }\end{array}$ & $7.0(5.0-7.75)$ & $10.7 \pm 6.34$ & 0.01 \\
\hline $\begin{array}{c}\text { Headache, migraine } \\
\text { pain* }\end{array}$ & $8.0(6.0-9.0)$ & $7.0(5.0-9.0)$ & 0.35 \\
\hline Vel & & & 0.32 \\
\hline
\end{tabular}

Values are presented as the median (IQR) or the mean $\pm \mathrm{SD}$. Boldface type indicates statistical significance.

* Pain was rated on a scale from 1 to 10 , with 10 representing the most severe pain.

whether these atypical pain syndromes are secondary to the cervical fusion of KFS as opposed to common comorbidities that are seen in KFS patients. For instance, our previous report using this data set showed that $93.3 \%$ of patients reported additional spine abnormalities, including scoliosis and degenerative disc disease. ${ }^{10}$ Other studies have also demonstrated an association between KFS and cervical myeloradiculopathy, potentially due to the hypermobility of cervical segments secondary to degenerative change. ${ }^{5,9,11}$ The possibility of spinal cord or nerve root compression may explain some of the types of pain that were described in this study, including numbness, tingling/pins and needles, and shooting pain. ${ }^{6}$

Patients with fusions at the occipitocervical or cervicothoracic junctions are generally expected to have greater disability. ${ }^{13,14}$ However, our findings suggest that participants with middle-level fusions, between $\mathrm{C} 2$ and C6, tended to report greater levels of overall muscle, joint, and nerve pain, although this did not reach statistical significance. This effect was not explained by the total number of cervical fusions or the severity of the fusions, as patients with middle cervical fusions tended to have fewer overall fusions and a smaller proportion of Samartzis type III fusions compared to participants with upper- or lower-level fusions. It is possible that KFS patients with middle-level cervical fusions are more susceptible to degenerative disease, disc herniation, myeloradiculopathy, or other spinous abnormalities that cause increased levels of pain. Indeed, prior studies have shown that patients with congenital cervical fusions have increased rates of degenerative change at the C3-4 level and a pattern of adjacent-segment degeneration, leading to cord compression specifically in the middle cervical region. The relative risk of degenerative cervical myelopathy in KFS patients is 3.3, suggesting that degenerative cervical myelopathy may play a significant role in the neurological symptoms and pain experienced by these patients. ${ }^{9}$ The propensity for degeneration may also explain why Samartzis type III fusions are associated with increased levels of joint and nerve pain; as described in prior studies, longer segments of fused vertebrae result in an increased lever arm and torque that are applied to free motion segments, increasing the probability of degeneration, subluxation, and instability adjacent to these fusions. ${ }^{5}$

The management of pain in KFS is generally conservative; surgery is reserved for patients with myelopathy or severe radiculopathy. ${ }^{6}$ Prior reports have suggested that between $6 \%$ and $43 \%$ of KFS patients require surgical intervention for neurological symptoms. ${ }^{8,13}$ In the present study, prescribed oral pain medications provided the most significant pain relief. Those participants who pursued surgery did not report significantly different levels of pain compared to those who received nonoperative management, but they did report increased levels of neurological symptoms and comorbidities. These neurological symptoms included dizziness, gait abnormalities, fine motor control difficulties, balance problems, and neuropathy. These findings are not surprising, given that the indications for surgical correction are severe myeloradiculopathy and not pain. The increased number of comorbidities in these patients, including spinal stenosis, disc herniation, and osteoarthritis, likely predisposed these individuals to vertebral hypermobility and resulting spinal cord or spinal root compression.

Our study has several limitations. First, due to the rarity of KFS, our statistical analyses are limited by a small sample size. Second, since we used patient-reported data, our measures of levels of fusion, pain, and pain relief are subject to reporting bias. Third, we are unable to account for variations in pain assessment across participants due to differences in the length of time since treatment or surgical intervention. Fourth, our cohort likely represents patients with more-severe presentations of KFS, as those individuals with no or mild symptoms may not yet have been diagnosed or may not be connected with the KFS Klippel-Feil Syndrome Freedom registry. Fifth, since we do not have access to radiographic images from these participants, we were unable to comment on modern cervical deformity parameters, such as T1 slope, cervical lordosis, cervical sagittal imbalance, and chin-brow angle. Description of such parameters in the KFS literature is limited; most reports on KFS have focused on lateral deformity/scoliosis rather than anteroposterior deformity. With regard to sagittal imbalance, a prior study by Xue et al. reported a mean sagittal cervical alignment of $29.9^{\circ}$ in a group of 28 patients..$^{15}$ It is possible that KFS patients with hemivertebral fusions or fusions at the craniocervical or cervicothoracic junction may be prone to greater alignment malformations; however, further research will be required to investigate this relationship, as well as its association with pain. Sixth, the surveys do not include follow-up data; as a result, our analyses are limited to a single time point, which would be the moment that the survey was completed. Finally, our conclusions are limited, as they are based on the nature of the survey questions-for example, pain relief afforded by "surgery" is not always further broken down into specific procedures or interventions, which disallows more nuanced analysis. 


\section{Conclusions}

Pain has not been well documented in the literature as one of the symptoms of KFS. In our analysis, we found that over $90 \%$ of participants experienced pain, primarily in the shoulders, upper back, and neck. Participants with an increased number of overall cervical fusions or multilevel, contiguous fusions reported greater levels of muscle, joint, and nerve pain. Participants who pursued surgery experienced more comorbidities and neurological symptoms, such as balance problems, gait disturbances, and fine motor control difficulties, but they did not report more significant pain than nonsurgically treated participants.

\section{Acknowledgments}

We wish to acknowledge CoRDS and Klippel-Feil Syndrome Freedom (KFS Freedom), which collected the data used in the present study. The KFS survey used in this study was written by Emily Lemiska, with review and guidance by Sharon Rose Nissley and Philip F. Giampietro, MD, PhD, on behalf of KFS Freedom. Dr. Giampietro is a Professor of Pediatrics and Division Director of Medical Genetics at Rutgers Biomedical and Health Sciences. Emily and Sharon Rose live with KFS.

\section{References}

1. Agarwal AK, Goel M, Bajpai J, Shukla S, Sachdeva N: Klippel Feil syndrome: a rare case report. J Orthop Case Rep 4:53-55, 2014

2. Brown MW, Templeton AW, Hodges FJ III: The incidence of acquired and congenital fusions in the cervical spine. Am J Roentgenol Radium Ther Nucl Med 92:1255-1259, 1964

3. Cho W, Lee DH, Auerbach JD, Sehn JK, Nabb CE, Riew KD: Cervical spinal cord dimensions and clinical outcomes in adults with Klippel-Feil syndrome: a comparison with matched controls. Global Spine J 4:217-222, 2014

4. Gruber J, Saleh A, Bakhsh W, Rubery PT, Mesfin A: The prevalence of Klippel-Feil syndrome: a computed tomography-based analysis of 2,917 patients. Spine Deform 6:448-453, 2018

5. Guille JT, Miller A, Bowen JR, Forlin E, Caro PA: The natural history of Klippel-Feil syndrome: clinical, roentgenographic, and magnetic resonance imaging findings at adulthood. J Pediatr Orthop 15:617-626, 1995

6. Kim JB, Park SW, Lee YS, Nam TK, Park YS, Kim YB: Two cases of Klippel-Feil syndrome with cervical myelopathy successfully treated by simple decompression without fixation. Korean J Spine 12:225-229, 2015

7. Menger RP, Notarianni C: Klippel Feil syndrome, in StatPearls. Treasure Island, FL: StatPearls Publishing, 2019 (https://www.ncbi.nlm.nih.gov/books/NBK493157/) [Accessed October 18, 2019]

8. Nagib MG, Maxwell RE, Chou SN: Identification and management of high-risk patients with Klippel-Feil syndrome. J Neurosurg 61:523-530, 1984

9. Nouri A, Martin AR, Lange SF, Kotter MRN, Mikulis DJ, Fehlings MG: Congenital cervical fusion as a risk factor for development of degenerative cervical myelopathy. World Neurosurg 100:531-539, 2017
10. Nouri A, Patel K, Evans H, Saleh M, Kotter MRN, Heary RF, et al: Demographics, presentation and symptoms of patients with Klippel-Feil syndrome: analysis of a global patientreported registry. Eur Spine J 28:2257-2265, 2019

11. Nouri A, Tetreault L, Zamorano JJ, Mohanty CB, Fehlings MG: Prevalence of Klippel-Feil syndrome in a surgical series of patients with cervical spondylotic myelopathy: analysis of the prospective, multicenter AOSpine North America Study. Global Spine J 5:294-299, 2015

12. Samartzis DD, Herman J, Lubicky JP, Shen FH: Classification of congenitally fused cervical patterns in Klippel-Feil patients: epidemiology and role in the development of cervical spine-related symptoms. Spine (Phila Pa 1976) 31:E798-E804, 2006

13. Theiss SM, Smith MD, Winter RB: The long-term followup of patients with Klippel-Feil syndrome and congenital scoliosis. Spine (Phila Pa 1976) 22:1219-1222, 1997

14. Vujasinovic Stupar N, Pavlov-Dolijanovic S, Hatib N, Banko B, Djukic M, Nikolic Jakoba N: Multiple major and minor anomalies associated with Klippel-Feil syndrome: a case report. Arch Rheumatol 31:82-86, 2015

15. Xue X, Shen J, Zhang J, Tian Y, Zhao H, Wang Y, et al: Klippel-Feil syndrome in congenital scoliosis. Spine (Phila Pa 1976) 39:E1353-E1358, 2014

\section{Disclosures}

Dr. Tessitore reports receiving training fees from Spineart, NuVasive, and DePuy Synthes.

\section{Author Contributions}

Conception and design: Nouri, Patel. Acquisition of data: Nouri, Patel. Analysis and interpretation of data: Nouri, Patel. Drafting the article: Patel. Critically revising the article: all authors. Reviewed submitted version of manuscript: all authors. Approved the final version of the manuscript on behalf of all authors: Nouri. Statistical analysis: Patel. Study supervision: Nouri, Cheng.

\section{Supplemental Information \\ Online-Only Content}

Supplemental material is available with the online version of the article.

Appendix 1: CoRDS Questionnaire. https://thejns.org/doi/ suppl/10.3171/2019.9.SPINE19820.

Appendix 2: KFS Freedom Questionnaire. https://thejns.org/ doi/suppl/10.3171/2019.9.SPINE19820.

\section{Previous Presentations}

Portions of this work were presented in oral form at the Global Spine Congress, Toronto, Ontario, Canada, May 17, 2019.

\section{Correspondence}

Aria Nouri: Hôpitaux Universitaires de Genève, University of Geneva, Switzerland. aria.nouri9@gmail.com. 